doi:10.1017/S0022215116007349

\section{ID: IP238}

Clinical Significance of Sensorineural

Hearing Loss in Pediatric Chronic Otitis

Media

Presenting Author: Noam Yehudai

Noam Yehudai, Michal Luntz

Bnai Zion Medical Center, Technion - The

Bruce Rappaport Faculty of Medicine, Haifa,

Israel

Learning Objectives: to promote awareness to the long term effects of chronic OM in terms of SNHL.

Introduction: Hearing loss is considered a common complication and sequela of chronic otitis media (COM). The loss is usually conductive, but sensorineural involvement also occurs. Clinically significant sensorineural hearing loss (SNHL) has been reported in adults with COM; however its significance in children is still unclear. The aim of the study is to assess the severity of SNHL in single sided COM, in a group of children, using the contra-lateral healthy ear as a control and to define risk factors for the development of SNHL in COM. Characterizing these risk factors will assist in better defining treatment indications for COM and thus reduce the occurrence of SNHL.

Methods: The study cohort included 124 children with unilateral COM operated between 1997 and 2010. Mean age at surgery was $13.3 \pm 3.2$ years (range, 7-18 years), and mean duration of disease was $88.4 \pm 45.0$ months (range, 6 -192 months). Bone conduction (BC) pure tone average (PTA) of the two ears was calculated as the average of BC thresholds at 500,1000, 2000 and $4000 \mathrm{~Hz}$. The parameters evaluated included: demographics (age, sex, and side), duration of disease, presence and location of cholesteatoma, previous otologic history and previous ear surgery.

Results: A statistically significant difference in BC-PTA was found between the diseased ear $(12.74 \pm 8.75 \mathrm{~dB})$ and the healthy ear $(9.36 \pm 6.33 \mathrm{~dB}) \quad(\mathrm{p}<0.01)$. Statistically significant correlation was found between the level of SNHL in the diseased ear, age and the presence of cholesteatoma.

Conclusions: One of the complications of chronic COM is the development of SNHL in addition to conductive hearing loss. It is imperative to actively treat COM patients in order to prevent the expected development of SNHL. An insistent approach to patients presenting with these factors is mandatory since they are prone to develop a more severe form of SNHL.

doi:10.1017/S0022215116007350

\section{ID: IP239}

Rat Mastoid Bullae Obliteration Using Hydroxyapatite/Chitosan Patch
Presenting Author: Keun-Ik Yi

Keun-Ik Yi

Pusan National University Hospital

Learning Objectives:

Purpose: Canal wall down mastoidectomy is often performed to prevent recurrence and complete removal of lesion in the treatment of otitis media with cholesteatoma. In this case, however, several problems can be caused to the mastoid cavity. Therefore to improve the long term stability of the mastoid cavity and to eliminate the cavity related problems significant modifications were introduced such as the mastoid obliteration technique using various methods including bone, cartilage, fat, flaps and others. This study evaluated the effectiveness of hydroxyapatite/ chitosan patch(HAp/Chi patch) known as the new materials to promote osteogenesis for mastoid obliteration in animal model.

Materials and Methods: Sprague-Dawley rats underwent mastoid obliteration using hydroxyapitie powder(Mimix ${ }^{\circledR}$ ), cartilage and HAp/Chi patch in each five ear. CT (computed tomography) and mastoid tissue sampling was done after twelve weeks. And we evaluated the degree of osteogenesis and inflammation of tissue.

Results: The mastoid cavity in HAp/Chi patch group was best filled in CT findings. In the histopathological aspects of the osteogenesis and inflammation, it exhibited 7.5\%/ $20 \%, 28 \% / 3 \%$ and $25 \% / 50 \%$ in the group of cartilage, hydroxyapitie powder and HAp/Chi patch group, respectively.

Conclusions: Hap/Chi patch is less absorbent, better to fill the mastoid cavity and more induce osteogenesis than the other obliteration material. But the degree of inflammation in Hap/Chi patch group is highest. It may be necessary to secure the stability of the mastoid obliteration material through additional experiments.

doi:10.1017/S0022215116007362

\section{ID: IP240}

\section{Facial Paralysis in Chronic Otitis Media} with Cholesteatoma

\section{Presenting Author: Nadir Yildirim}

Nadir Yildirim ${ }^{1}$, Sinan Aksoy ${ }^{2}$, Sermin Tok ${ }^{3}$

${ }^{1}$ Dumlupinar Universitesi Medical Faculty,

${ }^{2}$ DPU Medical Faculty Department of ORL-

HNS, ${ }^{3}$ Mersin University Department of

Radiology

\section{Learning Objectives:}

Objective: Facial paralysis is a rare and drastic complication of chronic otitis media and middle ear cholesteatoma. The predisposing factors that lead to facial nerve paralysis in 